\title{
Frequency of Monthly Ejaculation at Sexual Intercourse, DNA Fragmentation Index and Fertilization Rate among Sperm Donors and Fertility-Challenged Men in Sub-Saharan Africa: Paper 1
}

\author{
Abayomi B. Ajayi', Bamgboye M. Afolabi ${ }^{2 *}$, Victor D. Ajayi ${ }^{1}$, Ifeoluwa 0. Oyetunji ${ }^{1}$, \\ Adedamilola Atiba1, Seun Saanu'1, Ayodeji T. Adeoye1, Joy Ehichioya1, Ibukun I. Ayelehin'1 \\ ${ }^{1}$ Nordica Fertility Center, Lagos, Nigeria \\ ${ }^{2}$ Health, Environment and Development Foundation, Lagos, Nigeria \\ Email: ‘bmafolabi@gmail.com, heendef05@gmail.com
}

How to cite this paper: Ajayi, A.B., Afolabi, B.M., Ajayi, V.D., Oyetunji, I.O., Atiba, A., Saanu, S., Adeoye, A.T., Ehichioya, J. and Ayelehin, I.I. (2019) Frequency of Monthly Ejaculation at Sexual Intercourse, DNA Fragmentation Index and Fertilization Rate among Sperm Donors and Fertility-Challenged Men in Sub-Saharan Africa: Paper 1. Open Journal of Urology, 9, 181-194.

https://doi.org/10.4236/oju.2019.911021

Received: September 29, 2019

Accepted: November 24, 2019

Published: November 27, 2019

Copyright $\odot 2019$ by author(s) and Scientific Research Publishing Inc. This work is licensed under the Creative Commons Attribution International License (CC BY 4.0).

http://creativecommons.org/licenses/by/4.0/

\section{(c) (i) Open Access}

\begin{abstract}
Introduction: In humans, the ideal ejaculation frequency depends on age, exercise and sexual potential. Natural ejaculation frequency balances the testosterone levels in the body. Materials and Methods: Semi-structured questionnaire was used to collect information from subjects. Semen samples were collected from sperm donors and sub-fertile men who presented for infertility challenges. Processing and analysis of semen samples were done according to World Health Organization guidelines. Sperm DNA fragmentation was evaluated using the Halosperm ${ }^{\oplus}$ kit. Results: A total of 114 subjects, including 19 sperm donors and 95 sub-fertile males were studied. There was a significant difference $(\mathrm{t}=-5.96, \mathrm{P}$-value $=0.00001)$ in the mean $[ \pm \mathrm{sd}]$ age of sperm donors (30.8 [8.1]) and that of sub-fertile men (42.3 [76]). There was a significant difference $(\mathrm{t}=-4.10, \mathrm{P}$-value $=0.0005)$ in the mean monthly ejaculation during sexual intercourse (MESI) among sperm donors with DNA fragmentation index $<25 \%$ (3.0 [1.0]) compared to those with DNA fragmentation index $\geq 25 \%(9.3[5.5])$. There was also a significant difference $(t=-2.20$, P-value $=0.02)$ in MESI among sub-fertile men aged $\geq 40$ years (8.9 [4.6]) than among those aged $<40$ years (10.8 [3.8]). Entrepreneurs $(59.3 \%)$ and non-smokers (84.6\%) reported higher frequency (16 - 20) of MESI than others. There was a significant but negative correlation between frequency of MESI and DNA fragmentation index category (coef. $=-0.67, \mathrm{SE}=0.28, \mathrm{t}=$ $-2.40, \mathrm{P}$-value $=0.02,95 \% \mathrm{CI}:-1.24,-0.10)$. Conclusion: In men $<40$ years,
\end{abstract}


fewer MESI did not worsen the DFI. A higher number of professionals such as doctors, lawyers and engineers, reported lower monthly frequency of sexual ejaculations compared to entrepreneurs. Entrepreneurs and non-smokers had the highest frequencies of MESI.

\section{Keywords}

Monthly Sexual Ejaculation Rate, DNA Fragmentation Index, Male Infertility, Age Group, Body Mass Index, Social Habits, Black Africans

\section{Introduction}

In recent times, male infertility has become a global concern despite the clamor against population explosion. Male infertility is defined as a condition whereby a man has a problem with his reproductive system and cannot start a pregnancy with a female partner [1]. The American Pregnancy Organization reported that male infertility factors contribute to approximately $30 \%$ of all infertility cases and male infertility alone accounts for approximately one-fifth of all infertility cases [2]. Among the causes of male infertility are ejaculation problems which may include 1) premature ejaculation, 2) retrograde ejaculation, which occurs when semen is forced back into the bladder, 3) erection dysfunctions and 4) complication from radiation therapy or surgery.

The exact frequency of ejaculation before achieving pregnancy has not been defined probably because such frequency is not the main determinant of achieving pregnancy. However, frequency of ejaculation has been linked to seminal fluid parameter which is of paramount importance when investigating male infertility. The study of Mayorga-Torres et al. [3] shows that total sperm count and seminal volume per ejaculation declined and remained decreased for the duration of the daily ejaculation period. However, ejaculation control and discipline should not be confused with frequency of sex. Also, as medical evidence suggests that men experience a significant loss of zinc with each ejaculation, eating a food high in zinc after ejaculation is recommended.

The search for a cost effective fertility treatment has been important. Tesarik et al. [4] suggested that repeated ejaculations may improve sperm DNA quality which may facilitate better reproductive outcome [5] [6].

Hardly is there any study on monthly frequency of male ejaculation during sexual intercourse among sub-Sahara Black Africans relative to occupation, male infertility and DNA fragmentation index. An earlier study in Nigeria surveyed the effect of repeated semen ejaculation on sperm quality but did not delve into the fertility status or the occupation of the study subjects [7]. The objective of this study therefore was to determine the frequency of monthly ejaculation of sperm donors and sub-fertile men and report any possible association with age, occupation, type of infertility and DNA fragmentation index. 


\section{Materials and Methods}

This first paper involves materials and methods for harvesting data on MESI and DNA fragmentation index (DFI in \%). The second paper will focus on MESI and the fertilization rate among sperm donors and among sub-fertile men. In this first paper, a semi-structured questionnaire was designed to collect socio-demographic data; medical and surgical history; history, type and duration of infertility; miscarriage in partners and week of such miscarriage; undescended testes, testicular torsion, varicocele and childhood mumps; as well as history of .sexually transmitted illnesses. In addition, the questionnaire probed into frequency of monthly ejaculation, smoking and alcohol habits and use of medicinal herbs (agbo). Sperm was collected from each subject, whether donating sperm or consulting for male factor infertility, and processed according to WHO specification as described in our earlier paper [8]. Briefly, semen sample was collected by masturbation after a minimum/maximum of $2-7$ days of sexual abstinence and transported almost immediately to the laboratory for analysis. Each patient was counselled on 1) the need for accuracy in the collection of semen, 2) the kits (wide-mouth measuring cylinder) to be used for the collection and 3) the need to report any loss of semen during collection. Each patient has a medical record file with his data such as name, age (or date of birth), days of abstinence, date and time of collection, if there was any loss in semen volume during collection (incomplete collection) and the commencement of seminal fluid analysis in the laboratory. Conventional seminal fluid analysis was performed according to WHO guidelines [9] to detect semen volume, total sperm count, motility, and morphology as reported earlier [8]. The determination of human sperm DFI (\%) was reported in our earlier paper [10]. Briefly, SCD test was performed according to Fernandez et al., [11] using the Halosperm ${ }^{\circledast}$ kit (INDAS Laboratories, Madrid, Spain). After processing, slides were briefly washed in tap water and allowed to dry. This processing provided four dispersion patterns: sperms with 1) large halo: 2) moderate halo: 3) small halo: 4) Sperm with no halo. Sperm DFI (\%) was calculated as the proportion of sperm with big, small and no halos, to the total sperm count per slide. We assessed two slides for every patient, and a total of 1000 sperms were counted per slide. The halos correspond to relaxed DNA loops attached to the residual nuclear structure [11]. The spermatozoa without DNA fragmentation showed halos of dispersed DNA which can be large or medium, whereas those sperm nuclei with fragmented DNA produced either small halos or no halos at all. All the patients who came for sperm donation, who consulted for male infertility within the study period and who gave informed consent were included in the study except those who could not produce semen, those who declined consent and those whose information was inaccurate.

\section{Statistical Analysis}

Age was stratified into $<40$ and $\geq 40$ years old. The conventional classification of BMI as underweight (BMI < 18.5), normal weight (BMI 18.5 - 24.9), overweight 
(BMI 25.0 - 29.9) and obese (BMI $\geq 30$ ) was adopted. There was no underweight subject. All consenting men who presented for fertility assessment; those who were currently or within two weeks prior to analysis, not on any medication, particularly anabolic steroids, antibiotics and antimalarials, or any medication that would have interfered with spermatogenesis were included into the study. Frequency of monthly ejaculation at sexual intercourse (MESI) was classified as 1 for a frequency of $1-5,2$ for 6 - 10, 3 for 11 - 15 and 4 for 16 - 20. Sperm DNA fragmentation index (SDFI) was categorized as $<25 \%$ or good SDFI and $\geq 25 \%$ as bad SDFI. Statistical analysis was performed using STATA 13 for Windows (Stata Corps, College Station, Texas 77,845, USA). All variables were initially tested to determine variance homogeneity and data normality, and heteroscedastic data were transformed. Groups were compared using one-way ANOVA. Analyses carried out included frequency and percentage of proportions, appropriate bivariate (cross-tabulation) and multivariate regression analysis. Statistical variances between means were decided by Student's t-test when comparing 2 groups and by Kruskal-Wallis where comparing more than 2 groups. Outcomes were given as mean ( \pm standard deviation [sd]). The significance of differences between two or more than two proportions was determined using Chi-square $\left(\chi^{2}\right)$ test. Odds ratio was determined at 95\% Confidence Interval. Data were presented as figures, tables and graphs. Association between sperm DNA fragmentation index and continuous variables such as MESI was assessed using Linear Regression Analysis. Level of significance was set at $P<0.05$.

\section{Results}

This descriptive study conducted in 2018 was approved by the Nigerian Institute for Medical Research Institutional Review Board (NIMR IRB/18/007). Approximately $17 \%$ and $83 \%$ of the study subjects were sperm donors (SD) and non-donors (sub-fertile men, SFM) respectively. The mean [ \pm sd] age of the SFM (42.3 [7.6]) was significantly higher $(\mathrm{t}=-5.96, \mathrm{P}$-value $=0.00001)$ than that of the SD (40.4 [8.8]). Also, the mean [ $\pm \mathrm{sd}$ ] diastolic blood pressure (mm Hg) of the SFM (84.5 [11.6]) was significantly higher $(\mathrm{t}=-1.90, \mathrm{P}$-value $=0.03)$ than that of the SD (79.7 [9.7]). Further, SFM were approximately 1.5 times as likely to present with systolic hypertension compared to SD $\left(\chi^{2}=0.07, \mathrm{P}\right.$-value $=0.79$, $\mathrm{OR}=1.36,95 \% \mathrm{CI}: 0.45,4.11)$ and 6.5 time more likely to present with diastolic hypertension than SD $\left(\chi^{2}=5.86, \mathrm{P}\right.$-value $\left.=0.02, \mathrm{OR}=6.45,95 \% \mathrm{CI}: 1.41,29.52\right)$ (Data partially shown).

Of the 19 SD, 11 (57.9\%) were single and 8 (42.1\%) were married while of the 95 SFM, none was single. Majority $(8,42.1 \%)$ of the SD had technical occupation whereas most $(37,39.0 \%)$ of the SFM were entrepreneurs followed by professionals $(27,28.4 \%)$. Medical history showed that hypertension was most prevalent in both the SD $(4,21.1 \%)$ and SFM $(29,30.5 \%)$ while prevalence of sexually transmitted infection was higher $(21,22.1 \%)$ in the SFM than in SD $(1,5.3 \%)$. 
All the subjects with varicocele were sub-fertile men (Table 1).

Fertility profile of the SFM is as illustrated in Table 2 in which 91 (95.8\%) gave a history of infertility and only $4(4.2 \%)$ were not infertile. All the 39 $(100.0 \%)$ men aged $<40$ years presented with history of infertility compared to 52 (92.9\%) of those aged 40 years and above. All (100.0\%) the 29 SFM men with normal BMI, 37 (94.9\%) of those overweight and 25 (92.6\%) of those obese presented with history of infertility. Those aged $<40$ years were approximately 2.4 times as likely to have primary infertility compared to those aged $\geq 40$ years. Overweight SFM were 0.55 times as likely to present with primary infertility compared to normal weight men $\left(\chi^{2}=1.38, \mathrm{P}\right.$-value $=0.24, \mathrm{OR}=0.55,95 \% \mathrm{CI}=$ $0.21,1.48)$ while obese SFM were even less likely to present with primary infertility compared to normal weight men $\left(\chi^{2}=1.95, \mathrm{P}\right.$-value $=0.16, \mathrm{OR}=0.46,95 \%$ $\mathrm{CI}=0.15,1.37)$. Older SFM were about $3 \frac{1}{2} 2$ times more likely to have fathered a child than those aged $<40$ years $\left(\chi_{2}=6.65, \mathrm{P}\right.$-value $=0.01, \mathrm{OR}=3.36,95 \% \mathrm{CI}=$ $1.31,8.58)$. Compared to normal weight SFM, those overweight were 3.3 times more likely to have fathered a child $\left(\chi^{2}=4.34, \mathrm{P}\right.$-value $=0.04, \mathrm{OR}=3.34,95 \% \mathrm{CI}$ $=1.05,10.61)$ while obese men were approximately $4 \frac{1}{2}$ times more likely to have fathered a child $\left(\chi^{2}=6.01, \mathrm{P}\right.$-value $\left.=0.01, \mathrm{OR}=4.46,95 \% \mathrm{CI}=1.31,15.16\right)$. Overweight SFM were 0.95 as likely to have miscarriage in partner compared to normal weight men $\left(\chi^{2}=0.01, \mathrm{P}\right.$-value $\left.=0.92, \mathrm{OR}=0.95,95 \% \mathrm{CI}=0.34,2.69\right)$ while sub-fertile obese men were 1.3 times more likely to have miscarriage in partner compared to normal weight men $\left(\chi^{2}=0.23, \mathrm{P}\right.$-value $=0.63, \mathrm{OR}=1.31$, $95 \% \mathrm{CI}=0.44,3.86)$. Cumulatively, there was a marginally significant variation $\left(\chi^{2}=7.54, \mathrm{P}\right.$-value $\left.=0.05\right)$ in the frequency of ejaculation among sperm donors with DFI $<25 \%$ compared to DFI $\geq 25 \%$ (Table 3 ). Furthermore, the mean [ \pm sd] MESI of those with DFI < 25\% (3.0 [1.0]) was significantly lower ( $\mathrm{t}=$ -4.10 , P-value $=0.0005$ ) than those with DFI $\geq 25 \%$. Although there were significant differences in the proportion of sperm donors with different smoking habits $\left(\chi^{2}=8.26\right.$, P-value $\left.=0.04\right)$ and different drinking habits $\left(\chi^{2}=23.3\right.$, P-value $=0.001$ ), however there was no significant variation in the mean [ \pm sd] frequency of ejaculation of those with these social habits. Among the SFM, the overall mean [ \pm sd] frequency of MESI was 9.7 [4.4] (Table 4). The mean frequency of MESI among those aged $<40$ years (10.8 [3.8]) was significantly higher $(t=2.20$, $\mathrm{P}$-value $=0.02)$ than that of $\geq 40$ years. In all, $19(20.0 \%)$ sub-fertile men reported a frequency of 1 - 5 MESI while 29 (30.5\%), 34 (35.8\%) and 13 (13.7\%) reported frequencies of 6 -10,11-15 and 16 - 20. There was no significant difference in the mean $[ \pm \mathrm{sd}$ ] frequency of MESI relative to BMI groups or other variables. The frequency of MESI relative to occupations of the sub-fertile men is as illustrated in Figure 1. Approximately $54 \%$ of entrepreneurs, as against $23 \%$ of professional (medical doctors, lawyers and engineers) reported a frequency of 16 20 MESI. On the other side of the scale, approximately $37 \%$ of professionals, $31 \%$ of entrepreneurs and $21 \%$ of civil servants reported a frequency of $1-5$ MESI. The frequency of MESI among SFM with various social habits is as 
Table 1. Socio-demographic, medical and surgical characteristics of study subjects.

\begin{tabular}{|c|c|c|c|c|c|c|c|c|c|c|c|c|c|c|c|c|c|}
\hline \multirow{2}{*}{\multicolumn{2}{|c|}{ Variable }} & \multicolumn{4}{|c|}{ All } & \multicolumn{4}{|c|}{ Sperm Donor $(\mathrm{n}=19,16.7 \%)$} & \multicolumn{4}{|c|}{ Non-donor $(\mathrm{n}=95,83.3 \%)$} & \multirow{2}{*}{ t-test } & \multirow{2}{*}{$\mathrm{P}$-value } & \multirow{2}{*}{$x^{2}$} & \multirow{2}{*}{ P-value } \\
\hline & & Freq. & $\%$ & Mean & $\pm \mathrm{sd}$ & Freq. & $\%$ & Mean & $\pm s d$ & Freq. & $\%$ & Mean & $\pm \mathrm{sd}$ & & & & \\
\hline \multicolumn{2}{|c|}{$\mathrm{BMI}\left(\mathrm{Kg} / \mathrm{m}^{2}\right)$} & 114 & 100.0 & 27.5 & 4.7 & 19 & 16.7 & 26.2 & 4.4 & 95 & 83.3 & 27.7 & 4.7 & -1.34 & 0.10 & - & - \\
\hline \multirow{4}{*}{$\begin{array}{c}\text { Blood } \\
\text { Pressure } \\
(\mathrm{mm} \mathrm{Hg})\end{array}$} & Systolic & 114 & 100.0 & 130.1 & 20.4 & 19 & 16.7 & 127.8 & 17.9 & 95 & 83.3 & 130.6 & 20.9 & -0.60 & 0.28 & \multirow[b]{2}{*}{0.07} & \multirow[b]{2}{*}{0.79} \\
\hline & $\geq 140$ & 36 & 31.6 & 149.6 & 11.7 & 5 & 26.3 & 148.2 & 16.2 & 31 & 32.6 & 149.9 & 11.1 & -0.23 & 0.41 & & \\
\hline & Diastolic & 114 & 100.0 & 83.7 & 11.4 & 19 & 16.7 & 79.7 & 9.7 & 95 & 83.3 & 84.5 & 11.6 & -1.90 & 0.03 & & \\
\hline & $\geq 90$ & 43 & 37.7 & 95.1 & 8.0 & 2 & 10.5 & 100.0 & 0.0 & 41 & 43.2 & 94.9 & 8.1 & 4.03 & 0.00001 & 5.86 & 0.02 \\
\hline \multirow[b]{2}{*}{ Marital status } & Single & 11 & 9.7 & - & - & 11 & 57.9 & - & - & 0 & 0.0 & - & - & - & - & \multirow[b]{2}{*}{60.3} & \multirow[b]{2}{*}{0.00001} \\
\hline & Ever Married & 103 & 89.3 & - & - & 8 & 42.1 & - & - & 95 & 100.0 & - & - & - & - & & \\
\hline \multirow{9}{*}{ Occupation } & Professional & 30 & 26.3 & - & - & 3 & 15.8 & - & - & 27 & 28.4 & - & - & - & - & \multirow{9}{*}{32.4} & \multirow{9}{*}{0.00001} \\
\hline & Technical & 17 & 14.9 & - & - & 8 & 42.1 & - & - & 9 & 9.5 & - & - & - & - & & \\
\hline & Management & 6 & 5.3 & - & - & 0 & 0.0 & - & - & 6 & 6.3 & - & - & - & - & & \\
\hline & Entrepreneur & 42 & 36.8 & - & - & 5 & 26.3 & - & - & 37 & 39.0 & - & - & - & - & & \\
\hline & Civil servants & 9 & 7.9 & - & - & 0 & 0.0 & - & - & 9 & 9.5 & - & - & - & - & & \\
\hline & Farmer & 2 & 1.8 & - & - & 0 & 0.0 & - & - & 2 & 2.1 & - & - & - & - & & \\
\hline & Military & 3 & 2.6 & - & - & 0 & 0.0 & - & - & 3 & 3.2 & - & - & - & - & & \\
\hline & Students & 3 & 2.6 & - & - & 3 & 15.8 & - & - & 0 & 0.0 & - & - & - & - & & \\
\hline & Clergy & 2 & 1.8 & - & - & 0 & 0.0 & - & - & 2 & 2.1 & - & - & - & - & & \\
\hline \multirow{3}{*}{$\begin{array}{l}\text { Positive } \\
\text { Medical } \\
\text { History }\end{array}$} & Hypertension & 33 & 28.9 & - & - & 4 & 21.1 & - & - & 29 & 30.5 & - & - & - & - & \multirow{3}{*}{1.49} & \multirow{3}{*}{0.69} \\
\hline & $\begin{array}{c}\text { Other systemic } \\
\text { diseases }\end{array}$ & 1 & 0.9 & - & - & 0 & 0.0 & - & - & 1 & 1.1 & - & - & - & - & & \\
\hline & $\begin{array}{c}\text { Sexually } \\
\text { transmitted } \\
\text { infection }\end{array}$ & 22 & 19.3 & - & - & 1 & 5.3 & - & - & 21 & 22.1 & - & - & - & - & & \\
\hline \multirow{5}{*}{$\begin{array}{l}\text { Surgical } \\
\text { history }\end{array}$} & Groin surgery & 13 & 11.4 & - & - & 1 & 5.3 & - & - & 12 & 12.3 & - & - & - & - & \multirow{5}{*}{1.52} & \multirow{5}{*}{0.68} \\
\hline & $\begin{array}{l}\text { Testicular } \\
\text { torsion }\end{array}$ & 2 & 1.8 & - & - & 0 & 0.0 & - & - & 2 & 2.1 & - & - & - & - & & \\
\hline & $\begin{array}{l}\text { Childhood } \\
\text { mumps }\end{array}$ & 6 & 5.3 & - & - & 1 & 5.3 & - & - & 5 & 5.3 & - & - & - & - & & \\
\hline & $\begin{array}{l}\text { Undescended } \\
\text { testes }\end{array}$ & 0 & 0.0 & - & - & 0 & 0.0 & - & - & 0 & 0.0 & - & - & - & - & & \\
\hline & Varicocele & 7 & 6.1 & - & - & 0 & 0.0 & - & - & 7 & 7.4 & - & - & - & - & & \\
\hline
\end{tabular}

shown in Figure 2. Interestingly, those who never smoked cigarette had the highest frequency of 1 - 5 (79.0\%), 6 - 10 (82.8\%), $11-15$ (91.2\%) and $16-20$ (86.4\%) MESI. The frequency of 16 - 20 MESI was highest (53.9\%) among those who had never taken alcohol while it was lowest $(0.0 \%)$ among those who commonly drink alcohol. Age $<40$ years was responsible for a significant $25.54 \%$ variation observed in frequency of MESI $\left(\mathrm{R}^{2}=0.2554, \mathrm{~F}=2.91\right.$, $\mathrm{P}$-value $\left.=0.036\right)$ whereas age $\geq 40$ was responsible for an insignificant $8.67 \%$ variations observed $\left(\mathrm{R}^{2}=0.0867, \mathrm{~F}=1.21\right.$, P-value $\left.=0.318\right)$. 
Table 2. Fertility profile of sub-fertile men (non-donors) by age and BMI groups.

\begin{tabular}{|c|c|c|c|c|c|c|c|c|c|c|c|c|c|c|c|c|}
\hline \multirow{3}{*}{ Variable } & \multirow{3}{*}{\multicolumn{2}{|c|}{ Item }} & \multirow{3}{*}{ Mean } & \multirow{3}{*}{ $\pm s d$} & \multirow{3}{*}{ Freq. } & \multirow{3}{*}{$\%$} & \multicolumn{4}{|c|}{ Age (years) } & \multicolumn{6}{|c|}{$\operatorname{BMI}\left(\mathrm{Kg} / \mathrm{m}^{2}\right)$} \\
\hline & & & & & & & \multicolumn{2}{|c|}{$<40$} & \multicolumn{2}{|c|}{$\geq 40$} & \multicolumn{2}{|c|}{$18.5-24.9$} & \multicolumn{2}{|c|}{$25.0-29.9$} & \multicolumn{2}{|c|}{$\geq 30$} \\
\hline & & & & & & & Freq. & $\%$ & Freq. & $\%$ & Freq. & $\%$ & Freq. & $\%$ & Freq. & $\%$ \\
\hline \multirow{2}{*}{$\begin{array}{l}\text { History of } \\
\text { infertility }\end{array}$} & & Yes & - & & 91 & 95.8 & 39 & 100.0 & 52 & 92.9 & 29 & 100.0 & 37 & 94.9 & 25 & 92.6 \\
\hline & & No & - & & 4 & 4.2 & 0 & 0.0 & 4 & 7.1 & 0 & 0.0 & 2 & 5.1 & 2 & 7.4 \\
\hline \multicolumn{7}{|c|}{$\chi^{2}$ (P-value) } & \multicolumn{4}{|c|}{$2.88(0.09)$} & \multicolumn{2}{|c|}{ - } & \multicolumn{2}{|c|}{$1.51(0.22)$} & \multicolumn{2}{|c|}{$2.19(0.14)$} \\
\hline \multicolumn{7}{|c|}{ Odds Ratio (95\% Confidence Interval) } & \multicolumn{4}{|c|}{ undefined } & \multicolumn{2}{|c|}{-} & \multicolumn{2}{|c|}{ undefined } & \multicolumn{2}{|c|}{ undefined } \\
\hline Type of & & rimary & - & & 40 & 46.3 & 22 & 56.4 & 18 & 32.1 & 16 & 55.2 & 15 & 38.5 & 9 & 33.3 \\
\hline infertility & & condary & - & & 51 & 53.7 & 17 & 43.6 & 34 & 60.7 & 13 & 44.8 & 22 & 56.4 & 16 & 59.3 \\
\hline & & $\chi^{2}(\mathrm{P}-\mathrm{val}$ & & & & & & 4.25( & $.04)$ & & & & 1.38 & $.24)$ & 1.95 & $.16)$ \\
\hline & ds Rati & tio $(95 \%$ Con & fidence & Interv & & & & $.44(1.0$ & $4,5.73)$ & & & & $0.55(0$ & $1,1.48)$ & $0.46(0$ & , 1.37) \\
\hline & & 0 & 0.0 & 0.0 & 25 & 21.9 & 3 & 7.7 & 6 & 10.7 & 0 & 0.0 & 4 & 10.2 & 5 & 18.5 \\
\hline & & $1-3$ & 2.3 & 0.7 & 31 & 27.2 & 19 & 48.7 & 9 & 16.1 & 8 & 27.6 & 13 & 33.3 & 7 & 25.9 \\
\hline & & $4-6$ & 5.0 & 0.7 & 21 & 18.4 & 13 & 33.3 & 8 & 14.3 & 11 & 37.9 & 8 & 20.5 & 2 & 7.4 \\
\hline & & $7-9$ & 7.6 & 0.7 & 16 & 14.0 & 4 & 10.3 & 12 & 21.4 & 4 & 13.8 & 7 & 18.0 & 5 & 18.5 \\
\hline & & $\geq 10$ & 14.2 & 4.8 & 21 & 18.4 & 0 & 0.0 & 21 & 37.5 & 6 & 20.7 & 7 & 18.0 & 8 & 29.6 \\
\hline $\begin{array}{l}\text { Years trying } \\
\text { to conceive }\end{array}$ & & All & 6.2 & 5.3 & 95 & 100.0 & 39 & 41.1 & 56 & 58.2 & 29 & 30.5 & 39 & 41.1 & 27 & 28.4 \\
\hline & & $<40$ & 3.5 & 2.0 & 39 & 41.1 & - & - & - & - & - & - & - & - & - & - \\
\hline & 18 & $\geq 40$ & 8.1 & 6.1 & 56 & 58.2 & - & - & - & - & - & - & - & - & - & - \\
\hline & & $18.5-24.9$ & 6.8 & 5.3 & 29 & 30.5 & - & - & - & - & - & - & - & - & - & - \\
\hline & BMI & $25.0-29.9$ & 5.8 & 5.3 & 39 & 41.1 & - & - & - & - & - & - & - & - & - & - \\
\hline & & $\geq 30$ & 6.2 & 5.7 & 27 & 28.4 & - & - & - & - & - & - & - & - & - & - \\
\hline Ever fathered & & Yes & - & - & 34 & 35.8 & 8 & 20.5 & 26 & 46.4 & 5 & 17.2 & 16 & 41.0 & 13 & 48.2 \\
\hline a child & & No & - & - & 61 & 64.2 & 31 & 79.5 & 30 & 53.6 & 24 & 82.8 & 23 & 59.0 & 14 & 51.8 \\
\hline & & $\chi^{2}(\mathrm{P}-\mathrm{val}$ & & & & & & 6.65 & $0.01)$ & & & & 4.34 & .04) & 6.01 & $.01)$ \\
\hline & ds Rati & tio (95\% Con & fidence & Interv & & & & $.36(1.3$ & $1,8.58)$ & & & & $3.34(1$. & , 10.61) & $4.46(1$. & $15.16)$ \\
\hline Miscarriage & & Yes & - & - & 34 & 30.7 & 12 & 30.8 & 22 & 39.3 & 10 & 34.5 & 13 & 33.3 & 11 & 40.7 \\
\hline in partner & & No & - & - & 61 & 62.3 & 27 & 69.2 & 34 & 60.7 & 19 & 65.5 & 26 & 66.7 & 16 & 59.3 \\
\hline & & $\chi^{2}(\mathrm{P}-\mathrm{val}$ & & & & & & 0.72 & .40) & & & & 0.01 & $.92)$ & 0.23 & $.63)$ \\
\hline & As Rati & tio (95\% Con & fidence & Interv & & & & $.46(0.6$ & $1,3.46)$ & & & & $0.95(0$ & $4,2.69)$ & $1.31(0$ & , 3.86) \\
\hline & & 1 & - & & 22 & 64.7 & 9 & 75.0 & 13 & 59.1 & 7 & 70.0 & 6 & 46.2 & 9 & 81.8 \\
\hline $\begin{array}{l}\text { If "yes" how } \\
\text { many }\end{array}$ & & 2 & - & & 11 & 32.4 & 3 & 25.0 & 8 & 36.4 & 3 & 30.0 & 6 & 46.2 & 2 & 18.2 \\
\hline miscarriages & & 3 & - & & 0 & 0.0 & 0 & 0.0 & 0 & 0.0 & 0 & 0.0 & 0 & 0.0 & 0 & 0.0 \\
\hline & & 4 & - & & 1 & 2.9 & 0 & 0.0 & 1 & 4.5 & 0 & 0.0 & 1 & 7.6 & 0 & 0.0 \\
\hline
\end{tabular}


Table 3. Frequency of ejaculation among donors.

\begin{tabular}{|c|c|c|c|c|c|c|c|c|c|c|c|c|c|c|c|c|}
\hline & \multicolumn{14}{|c|}{ Frequency of ejaculation (per month) } \\
\hline & & & \multicolumn{2}{|c|}{$1-5$} & \multicolumn{2}{|c|}{$6-10$} & \multicolumn{2}{|c|}{$11-15$} & \multicolumn{2}{|c|}{$16-20$} & \multirow{2}{*}{$\chi^{2}$} & \multirow{2}{*}{ P-value } & \multirow{2}{*}{ Mean } & \multirow{2}{*}{ $\pm s d$} & \multirow{2}{*}{ t-test } & \multirow{2}{*}{$\mathrm{P}$-value } \\
\hline & & & Freq. & $\%$ & Freq. & $\%$ & Freq. & $\%$ & Freq. & $\%$ & & & & & & \\
\hline \multicolumn{3}{|c|}{ All } & 9 & 47.4 & 1 & 5.3 & 7 & 36.8 & 2 & 10.5 & - & - & 7.6 & 5.5 & - & - \\
\hline \multirow{2}{*}{\multicolumn{2}{|c|}{ Age }} & $<40$ & 8 & 88.9 & 1 & 100.0 & 5 & 71.4 & 2 & 100.0 & \multirow{2}{*}{1.57} & \multirow{2}{*}{0.67} & 7.4 & 5.6 & \multirow{2}{*}{-0.36} & \multirow{2}{*}{0.37} \\
\hline & & $\geq 40$ & 1 & 11.1 & 0 & 0.0 & 2 & 28.6 & 0 & 0.0 & & & 8.7 & 5.8 & & \\
\hline \multirow{3}{*}{\multicolumn{2}{|c|}{ BMI }} & $18.5-24.9$ & 4 & 44.5 & 0 & 0.0 & 4 & 57.1 & 2 & 100.0 & \multirow{3}{*}{5.49} & \multirow{3}{*}{0.48} & 9.2 & 5.6 & \multirow{3}{*}{$1.02^{*}$} & \multirow{3}{*}{0.38} \\
\hline & & $25.0-29.9$ & 3 & 33.3 & 1 & 100.0 & 1 & 14.3 & 0 & 0.0 & & & 5.0 & 4.8 & & \\
\hline & & $\geq 30$ & 2 & 22.2 & 0 & 0.0 & 2 & 28.6 & 0 & 0.0 & & & 7.0 & 5.8 & & \\
\hline \multirow{4}{*}{\multicolumn{2}{|c|}{ Occupation }} & Professional & 2 & 22.2 & 0 & 0.0 & 1 & 14.3 & 0 & 0.0 & \multirow{4}{*}{5.64} & \multirow{4}{*}{0.78} & 6.7 & 4.6 & \multirow{4}{*}{$0.80^{*}$} & \multirow{4}{*}{0.51} \\
\hline & & Technical & 2 & 22.2 & 1 & 100.0 & 3 & 42.9 & 2 & 100.0 & & & 9.9 & 5.8 & & \\
\hline & & Entrepreneur & 3 & 33.4 & 0 & 0.0 & 2 & 28.6 & 0 & 0.0 & & & 6.2 & 5.3 & & \\
\hline & & Student & 2 & 22.2 & 0 & 0.0 & 1 & 14.3 & 0 & 0.0 & & & 5.0 & 6.1 & & \\
\hline & FI & $<25$ & 5 & 55.6 & 0 & 0.0 & 0 & 0.0 & 0 & 0.0 & & $005-$ & 3.0 & 1.0 & 110 & 0005 \\
\hline & & $\geq 25$ & 4 & 44.4 & 1 & 100.0 & 7 & 100.0 & 2 & 100.0 & & & 9.3 & 5.5 & & \\
\hline & & Never & 9 & 100 & 0 & 0.0 & 5 & 71.4 & 2 & 100.0 & & & 7.1 & 5.7 & & \\
\hline & & 1 - 5 sticks daily & 0 & 0.0 & 1 & 100.0 & 2 & 28.6 & 0 & 0.0 & & & 10.7 & 2.3 & & \\
\hline & 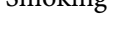 & 6 - 10 sticks daily & 0 & 0.0 & 0 & 0.0 & 0 & 0.0 & 0 & 0.0 & 0.20 & 0.04 & 0.0 & 0.0 & 1.11 & 0.01 \\
\hline Social & & $>10$ sticks daily & 0 & 0.0 & 0 & 0.0 & 0 & 0.0 & 0 & 0.0 & & & 0.0 & 0.0 & & \\
\hline habits & & Never & 6 & 66.7 & 0 & 0.0 & 2 & 28.6 & 0 & 0.0 & & & 5.0 & 4.4 & & \\
\hline & Alcohol & Rarely & 0 & 0.0 & 0 & 0.0 & 0 & 0.0 & 0 & 0.0 & & & 0.0 & 0.0 & & \\
\hline & intake & Occasionally & 3 & 33.3 & 0 & 0.0 & 5 & 71.4 & 2 & 100.0 & 20.0 & 0.001 & 9.7 & 5.8 & 1.00 & 0.20 \\
\hline & & Commonly & 0 & 0.0 & 1 & 100.0 & 0 & 0.0 & 0 & 0.0 & & & 8.0 & 0.0 & & \\
\hline & & Never & 6 & 66.7 & 1 & 100.0 & 5 & 71.4 & 1 & 50.0 & & & 7.6 & 5.2 & & \\
\hline & rbal & Rarely & 2 & 22.2 & 0 & 0.0 & 0 & 0.0 & 1 & 50.0 & & 16 & 6.7 & 8.1 & $0.00 *$ & 0.01 \\
\hline medic & ition use & Occasionally & 1 & 11.1 & 0 & 0.0 & 2 & 28.6 & 0 & 0.0 & 200 & - & 8.7 & 5.8 & o.t. & (1) \\
\hline & & Commonly & 0 & 0.0 & 0 & 0.0 & 0 & 0.0 & 0 & 0.0 & & & 0.0 & 0.0 & & \\
\hline
\end{tabular}

${ }^{*}$ F-statistics.

\section{Discussion}

We examined frequency of monthly ejaculation at sexual intercourse (MESI) and DNA fragmentation index (DFI) among sperm donors and sub-fertile men who presented at our clinic and investigated the role that age, body mass index and occupation may play in this dynamics. Free radicals, also known as Reactive Oxygen Species (ROS) are suspected to be involved in the dynamics of high or low frequency of MESI and DNA fragmentation. Reactive Oxygen Species are generated from the normal body metabolism of internal oxygen but may become excessive in various conditions such as toxic environment exposures. In excess, 
Table 4. Frequency of ejaculation among sub-fertile men.

\begin{tabular}{|c|c|c|c|c|c|c|c|c|c|c|c|c|c|c|c|}
\hline & \multicolumn{14}{|c|}{ Frequency of ejaculation (per month) } \\
\hline & & \multicolumn{2}{|c|}{$1-5$} & \multicolumn{2}{|c|}{$6-10$} & \multicolumn{2}{|c|}{$11-15$} & \multicolumn{2}{|c|}{$16-20$} & \multirow{2}{*}{$\chi^{2}$} & \multirow{2}{*}{ P-value } & \multirow{2}{*}{ Mean } & \multirow{2}{*}{ $\pm s d$} & \multirow{2}{*}{ t-test } & \multirow{2}{*}{$\mathrm{P}$-value } \\
\hline & & Freq. & $\%$ & Freq. & $\%$ & Freq. & $\%$ & Freq. & $\%$ & & & & & & \\
\hline \multicolumn{2}{|c|}{ All } & 19 & 20.0 & 29 & 30.5 & 34 & 35.8 & 13 & 13.7 & - & - & 9.7 & 4.4 & - & - \\
\hline \multirow{2}{*}{ Age } & $<40$ & 5 & 26.3 & 9 & 31.0 & 19 & 55.9 & 6 & 46.2 & \multirow{2}{*}{6.14} & \multirow{2}{*}{0.10} & 10.8 & 3.8 & \multirow{2}{*}{2.20} & \multirow{2}{*}{0.02} \\
\hline & $\geq 40$ & 14 & 73.7 & 20 & 69.0 & 15 & 44.1 & 7 & 53.8 & & & 8.9 & 4.6 & & \\
\hline \multirow{3}{*}{ BMI } & $18.5-24.9$ & 7 & 36.8 & 6 & 20.7 & 12 & 35.2 & 4 & 30.8 & & & 9.5 & 4.6 & & \\
\hline & $25.0-29.9$ & 7 & 36.8 & 16 & 55.2 & 11 & 32.4 & 5 & 38.4 & 3.99 & 0.68 & 9.4 & 4.3 & $0.24^{*}$ & 0.79 \\
\hline & $\geq 30$ & 5 & 26.4 & 7 & 24.1 & 11 & 32.4 & 4 & 30.8 & & & 10.1 & 4.4 & & \\
\hline & Professional & 7 & 36.8 & 8 & 27.6 & 9 & 26.5 & 3 & 23.1 & & & 9.1 & 4.2 & & \\
\hline & Technical & 2 & 10.5 & 4 & 13.8 & 2 & 5.9 & 1 & 7.7 & & & 8.8 & 5.7 & & \\
\hline & Management & 0 & 0.0 & 2 & 6.9 & 3 & 8.8 & 1 & 7.7 & & & 11.7 & 2.7 & & \\
\hline & Entrepreneur & 6 & 31.5 & 11 & 37.9 & 13 & 38.2 & 7 & 53.9 & & $0-57$ & 10.1 & 4.3 & & \\
\hline ce upationi & Civil servants & 4 & 21.1 & 1 & 3.5 & 4 & 11.8 & 0 & 0.0 & 17.17 & 0.07 & 7.4 & 4.6 & 1.10 & 0.07 \\
\hline & Farmer & 0 & 0.0 & 0 & 0.0 & 2 & 5.9 & 0 & 0.0 & & & 12.0 & 0.0 & & \\
\hline & Military & 0 & 0.0 & 1 & 3.5 & 1 & 2.9 & 1 & 7.7 & & & 13.3 & 6.1 & & \\
\hline & Clergy & 0 & 0.0 & 2 & 6.9 & 0 & 0.0 & 0 & 0.0 & & & 8.0 & 0.0 & & \\
\hline & $<1$ & 2 & 10.5 & 2 & 6.9 & 5 & 14.7 & 0 & 0.0 & & & 9.4 & 3.6 & & \\
\hline & $1-3$ & 3 & 15.8 & 6 & 20.7 & 14 & 41.2 & 5 & 38.5 & & & 11.1 & 3.5 & & \\
\hline Years trying to & $4-6$ & 2 & 10.5 & 10 & 34.5 & 7 & 20.6 & 2 & 15.4 & 19.13 & 0.09 & 9.8 & 3.9 & 2.30 & 0.06 \\
\hline & $7-9$ & 4 & 21.1 & 3 & 10.3 & 5 & 14.7 & 4 & 30.8 & & & 10.1 & 4.9 & & \\
\hline & $\geq 10$ & 8 & 42.1 & 8 & 27.6 & 3 & 8.8 & 2 & 15.4 & & & 7.4 & 5.1 & & \\
\hline & $<25$ & 5 & 26.3 & 16 & 55.2 & 12 & 35.3 & 6 & 46.1 & & & 9.9 & 4.2 & & \\
\hline 21 & $\geq 25$ & 14 & 73.7 & 13 & 44.8 & 22 & 64.7 & 7 & 53.9 & 1.00 & 0.20 & 9.5 & 4.5 & . & 0.00 \\
\hline Miscarriage in & Yes & 8 & 42.1 & 8 & 27.6 & 13 & 38.2 & 5 & 38.5 & 1. 21 & 072 & 9.6 & 4.5 & & 000 \\
\hline partner & No & 11 & 57.9 & 21 & 72.4 & 21 & 61.8 & 8 & 61.5 & 1.01 & 0.13 & 9.7 & 4.4 & -010 & 0.43 \\
\hline History of & Yes & 19 & 100.0 & 28 & 96.6 & 31 & 91.2 & 13 & 100.0 & 24 & $0=2$ & 9.6 & 4.4 & & \\
\hline infertility & No & 0 & 0.0 & 1 & 3.4 & 3 & 8.8 & 0 & 0.0 & 5.27 & 0.50 & 11.0 & 2.0 & 1.20 & 0.10 \\
\hline & None & 0 & 0.0 & 1 & 3.5 & 3 & 8.8 & 0 & 0.0 & & & 11.0 & 2.0 & & \\
\hline Type of infertility & Primary & 8 & 42.1 & 9 & 31.0 & 17 & 50.0 & 6 & 46.2 & 6.31 & 0.39 & 10.0 & 4.5 & 0.47 & 0.63 \\
\hline & Secondary & 11 & 57.9 & 19 & 65.5 & 14 & 41.2 & 7 & 53.8 & & & 9.3 & 4.4 & & \\
\hline Ever fathered a & Yes & 7 & 36.8 & 16 & 55.2 & 9 & 326.5 & 2 & 15.4 & 839 & 0.04 & 8.8 & 4.0 & -145 & 008 \\
\hline child & No & 12 & 63.2 & 13 & 44.8 & 25 & 73.5 & 11 & 84.6 & 0.37 & 0.04 & 10.1 & 4.5 & -1.45 & 0.00 \\
\hline & Never & 15 & 79.0 & 24 & 82.8 & 31 & 91.2 & 11 & 84.6 & & & 9.8 & 4.2 & & \\
\hline$c_{r}$ & 1 - 5 sticks daily & 2 & 10.5 & 3 & 10.3 & 3 & 8.8 & 1 & 7.7 & 71 & 067 & 9.0 & 4.6 & 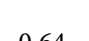 & $=0$ \\
\hline SIIOKMII & 6 - 10 sticks daily & 1 & 5.3 & 0 & 0.0 & 0 & 0.0 & 0 & 0.0 & 0.11 & $0.0 \%$ & 4.0 & 0.0 & 0.04 & 0.39 \\
\hline Social & $>10$ sticks daily & 1 & 5.3 & 2 & 6.9 & 0 & 0.0 & 1 & 7.7 & & & 9.8 & 7.2 & & \\
\hline habits & Never & 7 & 36.8 & 12 & 41.4 & 10 & 29.4 & 7 & 53.9 & & & 9.9 & 4.5 & & \\
\hline Alcohol & Rarely & 2 & 10.5 & 0 & 0.0 & 5 & 14.7 & 1 & 7.7 & 1079 & 029 & 9.9 & 5.4 & 14 & 94 \\
\hline intake & Occasionally & 9 & 47.4 & 11 & 37.9 & 12 & 35.3 & 5 & 38.5 & 10.13 & 0.23 & 9.3 & 4.7 & 0.14 & 0.04 \\
\hline & Commonly & 1 & 5.3 & 6 & 20.7 & 7 & 20.6 & 0 & 0.0 & & & 9.7 & 2.6 & & \\
\hline & Never & 11 & 57.9 & 2 & 72.4 & 25 & 73.5 & 8 & 61.5 & & & 9.8 & 4.1 & & \\
\hline Herbal medication & Rarely & 2 & 10.5 & 0 & 0.0 & 3 & 8.8 & 0 & 0.0 & 1765 & 004 & 12.0 & 5.7 & 0 & 7? \\
\hline use & Occasionally & 6 & 31.6 & 7 & 24.1 & 6 & 17.7 & 4 & 30.8 & 17.65 & 0.04 & 9.3 & 5.1 & 0.45 & 0.12 \\
\hline & Commonly & 0 & 0.0 & 1 & 3.5 & 0 & 0.0 & 1 & 7.7 & & & 8.2 & 5.4 & & \\
\hline
\end{tabular}




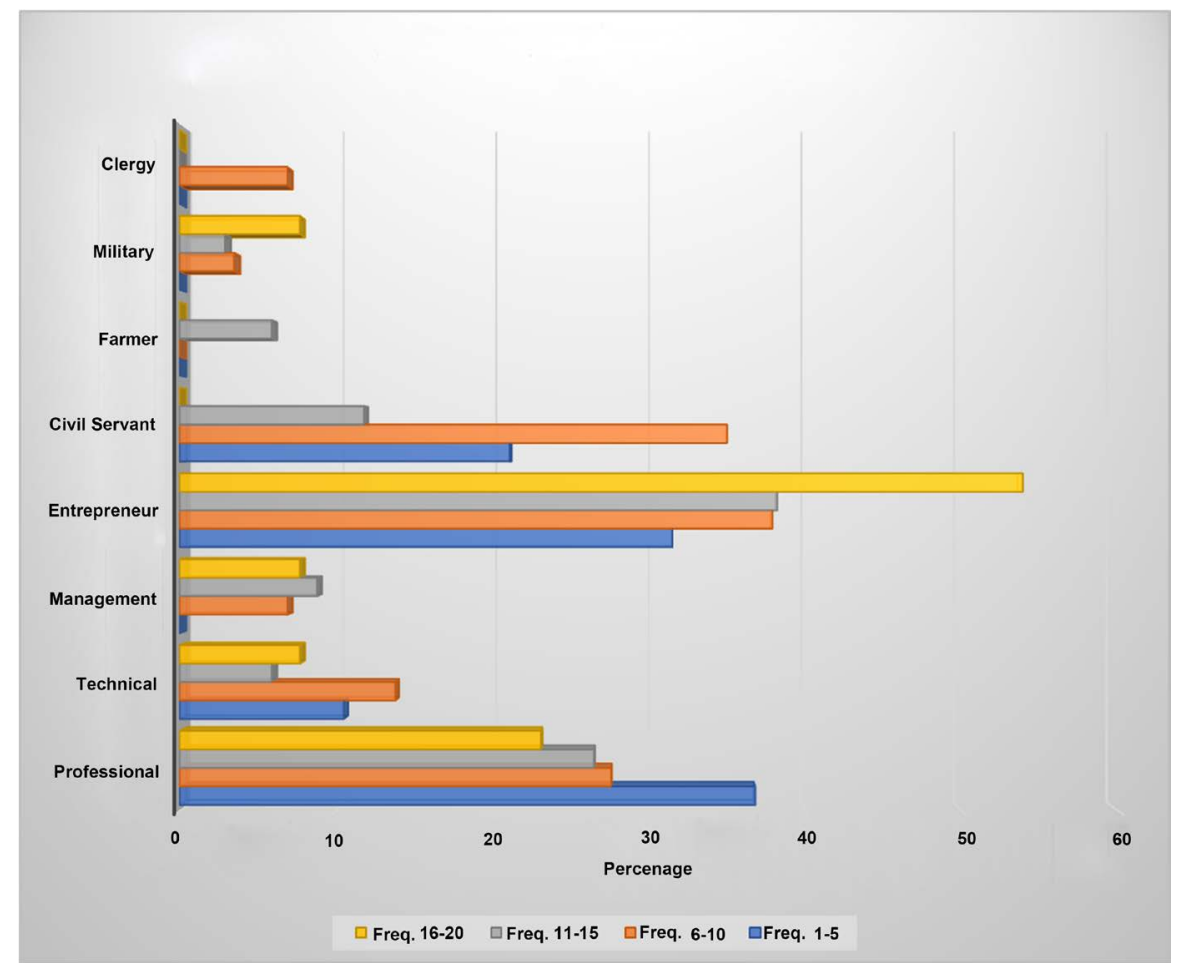

Figure 1. Frequency of monthly ejaculation during sexual intercourse among sub-fertile men in various occupational groups.

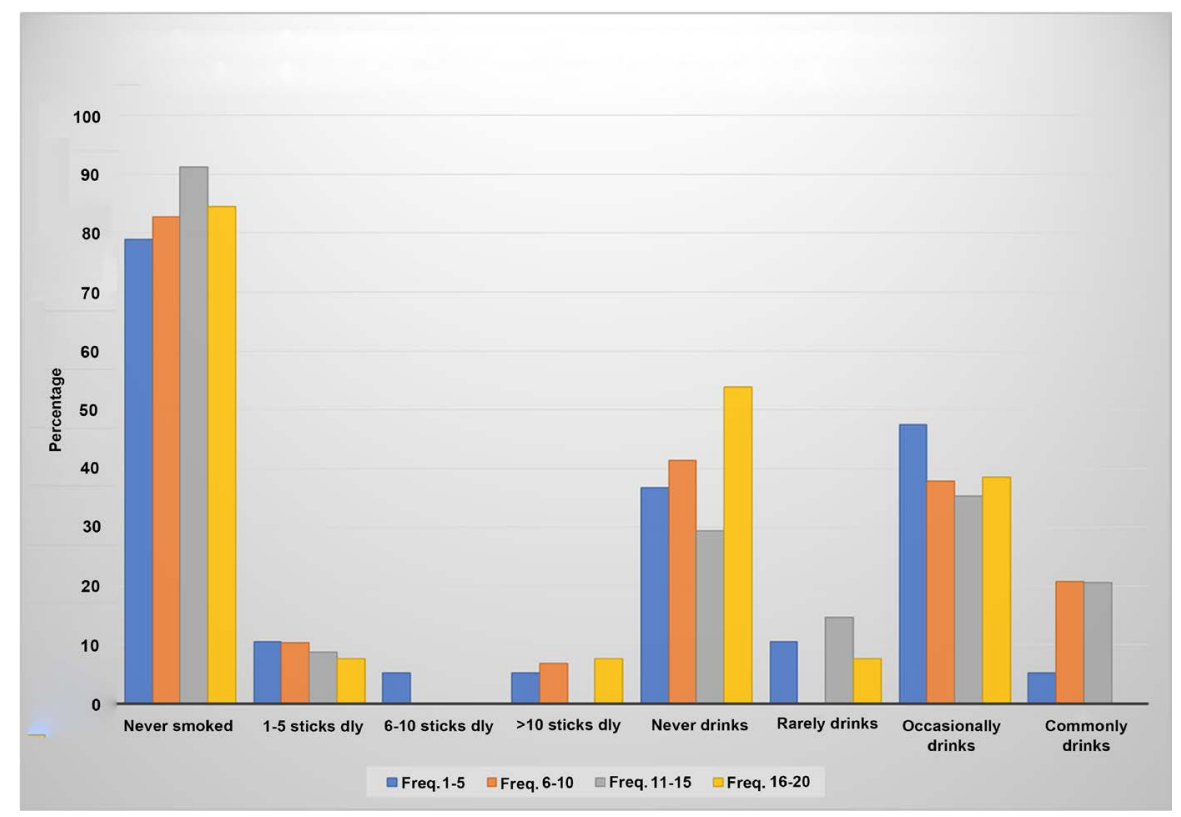

Figure 2. Frequency of monthly ejaculation during sexual intercourse among sub-fertie men with various social habits.

ROS do significant damages to cell structure, especially the deoxyribonucleic acid (DNA) within the nucleus of the cells. Although the epididymis, which stores sperms produced in the testicles, the testicles and semen, are equipped with abundant supply of antioxidants, which neutralizes ROS, this defense can be rup- 
tured when an imbalance occurs between ROS and antioxidant concentrations [12] [13] [14].

Consistent with previous findings in Nigeria [15] and elsewhere [16], our results suggest that systolic and diastolic hypertension are prevalent among men with fertility challenge. Among sub-fertile men, only about 23\% professionals reported high frequency of MESI (16 - 20) compared to about 54\% of entrepreneurs while no sub-fertile man who commonly drinks reported high frequency of MESI. To buttress these findings, Papaefstathiou et al. [17] reported that, among males, personal burnout, hypertension, and alcohol consumption correlated independently with erectile dysfunction $(p=0.001)$ and reduced total satisfaction $(p<0.001)$. Occupational stress such as abusive, aggressive, spiteful, incompetent, or unfair boss among professionals more than among entrepreneurs, might have a direct or indirect link to MESI. Of particular interest is that the proportion of sub-fertile males who never smoked cigarette was higher in each category of MESI. This may be a novel finding in sub-Sahara Africa. Possibly, chemicals from inhaled cigarette smoke damages penile blood vessels to induce poor erectile health and erection may not occur because damaged penile arteries cannot fill with blood after receiving signals from nerves in the penis [18]. More study is required to elucidate this phenomenon. In the same vein, those who never used herbal medication recorded higher proportions of MESI that those who rarely, occasionally or who frequently used herbal medications. A key finding is that age $<40$ years, negatively but significantly correlated with DNA fragmentation category whereas such phenomenon was not observed in the older age group. This finding is consisted with what Belloc et al. [18] reported that DNA fragmentation level is related to sperm motility and paternal age. Approximately 75\% of sub-fertile men with minimal (1 - 5) frequency of monthly ejaculation at sexual intercourse had a DNA fragmentation index of $>25 \%$. On the other hand, about $45 \%$ sperm donors with minimal ( 1 - 5) MESI had a DNA fragmentation index of $>25 \%$. Since the sperm donor were significantly younger than the sub-fertile men, advanced age may be the deciding factor in the DNA fragmentation, in consonance with the findings in several other studies conducted outside of Africa [19] [20] [21] [22] [23].

\section{Conclusion and Recommendations}

To conclude, we studied sperm donors and sub-fertile men presenting at our clinic for infertility problems and found that sperm donors were significantly younger than the sub-fertile men, that among sperm donors, mean $( \pm s d)$ frequency of MESI was 7.6 (5.5) per month compared to 9.7 (4.4) per month among sub-fertile men and that sub-fertile men aged $<40$ had a significantly higher MESI of 10.8 (3.8) per month compared to sub-fertile men with 8.9 (4.6) per month. Professionals, probably due to stress had low frequency of ejaculation than entrepreneurs, those who never smoked cigarette or those who never took alcohol had higher frequencies of monthly ejaculation than those who ever 
smoked cigarette or ever took alcohol. DNA fragmentation index was significantly but negatively correlated with age. Be that as it may, frequency of ejaculation is not the main determinant of achieving pregnancy though, it could contribute. The data in this study could be better evaluated by establishing an association with pregnancy outcome.

\section{Strengths and Weaknesses}

This study has some strengths and weaknesses. Concerning strength of the study, semen collection was very accurate and laboratory analysis of seminal fluid and DNA fragmentation was conducted using modern equipment and according to the latest WHO standard. However, the sample size was small and there could have been a bias in sampling. The study subjects were not representative of the general population, thus the data should not be extended to the generality. Further, the study was facility-based and does not reflect the frequency of monthly ejaculation at sexual intercourse of normal healthy males in the community. Furthermore, there were no underweight men with BMI $<18.5$ $\mathrm{kg} / \mathrm{m}^{2}$ in the study which could have made the data more robust.

\section{Acknowledgements}

Special thanks go to our patients.

\section{Funding}

This study did not receive funding from any source

\section{Conflicts of Interest}

The authors declare no conflicts of interest regarding the publication of this paper.

\section{References}

[1] https://www.stanfordchildrens.org

[2] https://www.americanpregnancy.org

[3] Mayorga-Torres, B.J., Cardona-Maya, W., Cadavid, A. and Camargo, M. (2013) Evaluation of Sperm Functional Parameters in Normozoospermic Infertile Individuals. Actas Urologicas Espanolas, 37, 221-227. https://doi.org/10.1016/j.acuro.2012.06.008

[4] Tesarik, J., Greco, E., Cohen-Bacrie, P. and Mendoza, C. (1998) Germ Cell Apoptosis in Men with Complete and Incomplete Spermiogenesis Failure. Molecular Human Reproduction, 4, 757-762. https://doi.org/10.1093/molehr/4.8.757

[5] Sanchez-Martin, P., Sanchez-Martin, F., Gonzalez-Martinez, M. and Gosalvez, J. (2013) Increased Pregnancy after Reduced Male Abstinence. Systems Biology in Reproductive Medicine, 59, 256-260. https://doi.org/10.3109/19396368.2013.790919

[6] Gosálvez, J., González-Martínez, M., López-Fernández, C., Fernández, J.L. and Sánchez-Martín, P. (2011) Shorter Abstinence Decreases Sperm Deoxyribonucleic Acid Fragmentation in Ejaculate. Fertility and Sterility, 96, 1083-1086. 
https://doi.org/10.1016/j.fertnstert.2011.08.027

[7] Nnatu, S., Giwa-Osagie, O. and Essien, E. (1990) Effect of Repeated Semen Ejaculation on Sperm Quality. Clinical and Experimental Obstetrics and Gynecology, 18, $39-42$.

[8] Ajayi, A.B., Afolabi, B.M., Victor, D.A., Oyetunji, I., et al. (2018) Semen Parameters Associated with Male Infertility in a Sub-Saharan Black Population: The Effect of Age and Body Mass Index. Journal of Gynecology and Infertility, 1, 1-8

[9] World Health Organization (2011) WHO Laboratory Manual for the Examination and Processing of Human Semen. 5th Edition, World Health Organization, Geneva.

[10] Ajayi, A.B., Afolabi, B.M., Ajayi, V.D., Oyetunji, I.O., Atiba, A., Saanu, S., Adeoye, A.T., Adeshida, T.E., Ehichioya, J. and Ayelehin, I.I. (2018) Evaluation of Sperm DNA Fragmentation amongst Infertile Black Africans. A Nigerian Study. Open Journal of Urology, 8, 297-316. https://doi.org/10.4236/oju.2018.811034

[11] Fernández, J.L., Muriel, L., Rivero, M.T., Goyanes, V., Vazquez, R. and Alvarez, J.G. (2003) The Sperm Chromatin Dispersion Test: A Simple Method for the Determination of Sperm DNA Fragmentation. Journal of Andrology, 24, 59-66.

[12] Aitken, J., Krausz, C. and Buckingham, D. (1994) Relationships between Biochemical Markers for Residual Sperm Cytoplasm, Reactive Oxygen Species Generation, and the Presence of Leukocytes and Precursor Germ Cells in Human Sperm Suspensions. Molecular Reproduction and Development, 39, 268-279. https://doi.org/10.1002/mrd.1080390304

[13] Greco, E., Scarselli, F., Iacobelli, M., Rienzi, L., Ubaldi, F., Ferrero, S., et al. (2005) Efficient Treatment of Infertility Due to Sperm DNA damage by ICSI with Testicular Spermatozoa. Human Reproduction, 20, 226-230.

https://doi.org/10.1093/humrep/deh590

[14] Moskovtsev, S.I., Jarvi, K., Mullen, J.B., Cadesky, K.I., Hannam, T. and Lo, K.C. (2010) Testicular Spermatozoa Have Statistically Significantly Lower DNA Damage Compared with Ejaculated Spermatozoa in Patients with Unsuccessful Oral Antioxidant Treatment. Fertility and Sterility, 93, 1142-1146.

https://doi.org/10.1016/j.fertnstert.2008.11.005

[15] Garko, B., Ogunsina, M.O. and Danbauchi, S.S. (2005) Sexual Dysfunction in Hypertensive Patients: Implications for Therapy. Annals of African Medicine, 4, 46-51.

[16] Guo, D., Shufeng, L., Behr, B. and Eisenberg, M. (2017) Hypertension and Male Fertility. The World Journal of Men's Health, 35, 59-64.

https://doi.org/10.5534/wjmh.2017.35.2.59

[17] Papaefstathiou, E.F., Apostolopoulou, A., Papaefstathiou, E.R., Moysidis, K., Hatzimouratidis, K. and Sarafis, P. (2019) The Impact of Burnout and Occupational Stress on Sexual Function in Both Male and Female Individuals: A Cross-Sectional Study. International Journal of Impotence Research.

https://doi.org/10.1038/s41443-019-0170-7

[18] Belloc, S., Benkhalifa, M., Cohen-Bacrie, M., Dalleac, A., Amar, E. and Zini, A. (2014) Sperm Deoxyribonucleic Damage in Normzoospermic Men Is Related to Age and Sperm Progressive Motility. Fertility and Sterility, 101, 1588-1593. https://doi.org/10.1016/j.fertnstert.2014.02.006

[19] Moskovtsev, S.I., Willis, J. and Mullen, J.B. (2006) Age-Related Decline in Sperm Deoxyribonucleic Acid Integrity in Patients Evaluated for Male Infertility. Fertility and Sterility, 85, 496-499. https://doi.org/10.1016/j.fertnstert.2005.05.075

[20] Barroso, G., Morshedi, M. and Oehninger, S. (2000) Analysis of DNA Fragmenta- 
tion, Plasma Membrane Translocation of Phosphatidylserine and Oxidative Stress in Human Spermatozoa. Human Reproduction, 15, 1338-1344.

https://doi.org/10.1093/humrep/15.6.1338

[21] Brinkworth, M.H. and Schmid, T.E. (2003) Effect of Age on Testicular Germ Cell Apoptosis and Sperm Aneuploidy in MF-1 Mice. Teratogenesis, Carcinogenesis, and Mutagenesis, 23, 103-109. https://doi.org/10.1002/tcm.10085

[22] Singh, N.P., Muller, C.H. and Berger, R.E. (2003) Effects of Age on DNA Double-Strand Breaks and Apoptosis in Human Sperm. Fertility and Sterility, 80, 1420-1430. https://doi.org/10.1016/j.fertnstert.2003.04.002

[23] Spano, M., Bonde, J.P., Hjollund, H.I., Kolstad, H.A., Cordelli, E. and Leter, G. (2000) Sperm Chromatindamage Impairs Human Fertility. The Danish First Pregnancy Planner Study Team. Fertility and Sterility, 73, 43-50.

https://doi.org/10.1016/S0015-0282(99)00462-8

\section{Abbreviations}

ANOVA-Analysis of variance

BMI-Body Mass Index

CI-Confidence interval

DFI-DNA fragmentation index

DNA-Deoxyribonucleic acid

MESI-Monthly ejaculation at sexual intercourse

NFC-Nordica Fertility Center

OR-Odds ratio

ROS-Reactive oxygen species

SD-Sperm donors

sd-Standard deviation

SFM - Sub-fertile men

TTC-Years trying to conceive

WHO-World Health Organization 